\title{
OBSERVATIONAL STUDIES OF THE STRUCTURE OF ACTIVE GALACTIC NUCLEI AND QSOs
}

\author{
J. S. MILLER \\ Lick Observatory, Board of Studies in Astronomy and Astrophysics \\ University of California, Santa Cruz, CA 95064
}

\section{Introduction}

Direct observational information on the structures of the central objects of active galaxies and quasi-stellar objects is hard to come by. Most of what we believe about these regions is inferred from data concerning the emission line spectra, the spectral energy distributions of the featureless continuum, and the luminosities and variability of the active nucleus. Data on the polarization of light from active galaxies, especially spectropolarimetric data which distinguishes between lines and continua, can offer additional insight into the nature of the central object, to some extent more direct information insofar as it can provide geometrical as well as physical information.

For some years I and my colleagues - at various times G. Schmidt, R. Antonucci, R. Goodrich, and L. Kay - have been engaged in a systematic program of spectropolarimetry of active galaxies and QSOs using the Lick Observatory 3-m Shane telescope equipped with an efficient polarimeter (Miller, Robinson and Schmidt 1980; Miller, Robinson and Goodrich 1988). The research involves large amounts of observing time, because the polarizations of the objects are usually small (at most a few percent) and many of the objects are faint. To date we have concentrated on Seyfert galaxies, which will be the primary subject of this paper, but we also have obtained considerable data on QSOs, optically violently variable quasars, and radio galaxies.

Polarization can arise as the result of several processes. It can be produced by the radiation process itself, as in synchrotron radiation. Scattering by dust polarizes light by two mechanisms: passage of light through aligned dust grains where we observe the transmitted light, or light scattering in a reflection nebula where we observe the scattered light. A third process is electron scattering, which is wavelength independent and can broaden emission lines. Often two or more polaragenic mechanisms can be operating, which confuses interpretation. In addition, interstellar polarization in our galaxy and in the host galaxy of the active nucleus may be present, and dilution of polarized light by an additional, unpolarized source such as stars, may further complicate interpretation. Thus it is often unclear what the nature of the polarizing mechanism is. Nevertheless, the data provide valuable insights even when the cause of polarization cannot be determined with much confidence. 


\section{Seyfert Galaxies}

While a variety of polarization characteristics of Seyfert nuclei have been observed, some systematic behaviour of considerable importance has been found. Most Seyferts do not have significant optical polarization, that is, polarization that is higher than that which might be expected from interstellar polarization. Among the intrinsically polarized Seyfert nuclei, it has been found (Schmidt and Miller 1980; Miller and Antonucci 1983; Antonucci 1983) that type 1's and 2's have different polarization characteristics: the type 1's tend to have low polarization $(\sim 1 \%)$ with the optical polarization position direction aligned with the radio structure, while the type 2 's can have higher polarizations (up to $\sim 20 \%$ ) with the polarization direction perpendicular to the radio structure. (Quasars, which have spectra similar to type 1 Seyferts, also show a parallel relationship between polarization and radio structure.)

We (Miller and Antonucci 1983) proposed a simple picture which explains these differences between type 1's and 2's: type 1 nuclei could contain a thin disk of material surrounding the central object, while type 2's could have a thick disk. In type 1's the disk does not block our direct view of the continuum-producing regions, and the small amount of polarization present is produced by scattering of light from the central region by material near the surface of the disk. The thick disk of the type 2's can block our direct view of the central regions, which are only visible as a result of light scattering by material above and below the disk in the general vicinity of the axis of the disk. If we further assume that the radio axis is aligned with the axis of the disk, which of course has been the standard picture for some time, then the polarization-radio structure relationships described above are explained. This is obviously a picture developed entirely from geometrical considerations, but, as we shall see, it is consistent with a large amount of data both geometrical and physical.

\subsection{SEYFERT 2 GALAXIES}

Our data on NGC 1068 first suggested to us the thick disk interpretation of the polarization (Miller and Antonucci 1983). When corrected for dilution by unpolarized starlight, the nuclear featureless continuum was found to have wavelength-independent polarization of about $16 \%$. We proposed electrons as the scattering particles.

This picture was extended with the discovery that the nucleus of NGC 1068 also contains a Seyfert type 1 region hidden from our direct view (Antonucci and Miller 1985). As Figure 1 shows, the spectrum of the total flux is that of a type 2 Seyfert galaxy, while the polarized flux spectrum closely resembles that of a type 1 , showing broad permitted lines as well as Fe II emission. Our picture was that the electrons located above and below the thick disk scatter both the featureless continuum and light from a high density line-emitting region.

Further confirmation of the obscuring disk or torus picture was obtained with a new device we constructed, an imaging polarimeter. This device allows the determination of the linear polarization of light in a direct image covering a field $30^{\prime \prime} \times 30^{\prime \prime}$. The obscuring torus picture suggests that light from the central object should illuminate the surrounding galaxy in two general directions more or less along the axis of the torus. Any dust present in the galaxy along these directions could reflect the nuclear light to us, while other directions should show no nuclear reflected light. The imaging polarimetry data shows just that (Miller, Goodrich and Mathews 1988): several arcsec- 
onds in direction roughly north-south from the nucleus, along the inferred torus axis, we detected polarized light with polarization position angles at right angles to the direction toward the nucleus. In addition we detected a small region $5^{\prime \prime}$ north-east of the nucleus which also is substantially polarized at right angles to the nuclear direction. Subsequent spectropolarimetry of this small region, which we call the "north-east knot," and the north-south strip or beam of light, shows that the polarization in all these regions rides rapidly to the blue; in the north-east knot it reaches $30 \%$ in the ultraviolet. This indicates that the light is reflected or scattered by dust grains in the NGC 1068 galaxy.

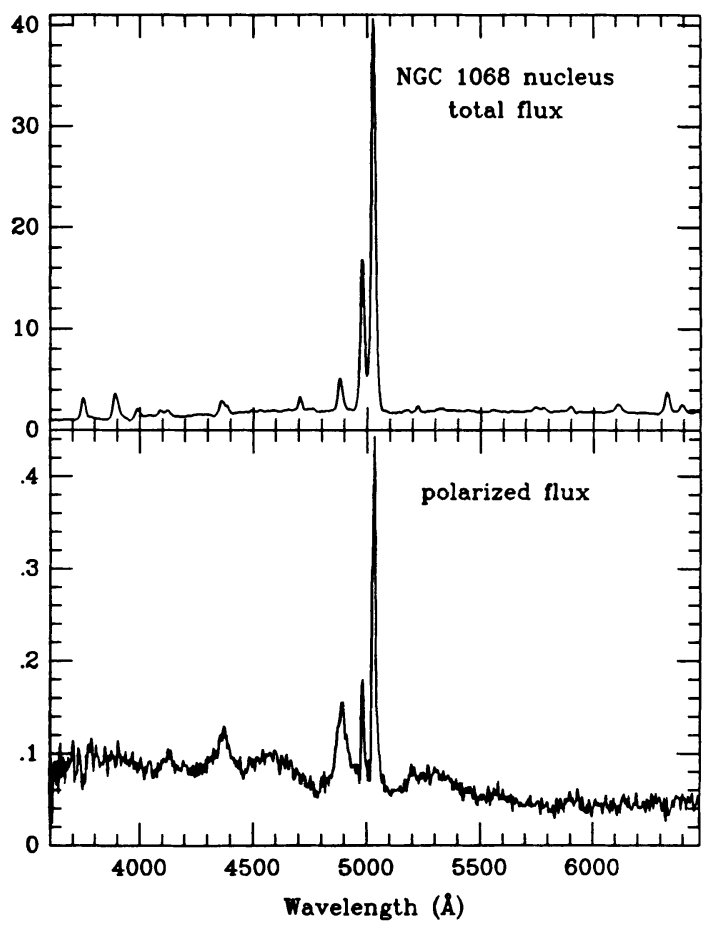

Figure 1 The total flux spectrum and polarized flux spectrum of the nucleus of NGC 1068.

That this extended polarized light is reflected nuclear light is confirmed by the spectrum of the polarized flux. Figure 2 shows the flux and polarized flux spectra of the north-east knot. As in the nuclear observations, the polarized flux from the knot has the spectrum of a Seyfert type 1 galaxy. Similarly, the polarized flux spectra of the north-south beam is that of a type 1 . Thus for the first time we are getting views of a Seyfert galaxy from different directions. From our vantage point NGC 1068 appears to be a Seyfert 2 galaxy, but if we were observing from a position in the north-east knot, we would call it a Seyfert 1 galaxy. 
There is an important difference between the polarized flux spectrum of the nuclear and that of the knot: the broad $\mathrm{H} \beta$ line is narrower in the knot spectrum than in the nucleus one. The full-width at half intensity is about $3000 \mathrm{~km} / \mathrm{sec}$ in the nuclear polarized flux and about $2200 \mathrm{~km} / \mathrm{sec}$ in the knot; similar narrower widths were observed in the north-south strip. Further, the nuclear spectrum has $\mathrm{H} \beta$ redshifted about $600 \mathrm{~km} / \mathrm{sec}$ with respect to the [O III] lines, while the off-nucleus regions show no difference in velocity. This is to be expected in the picture we have proposed, as the electron-scattered nuclear $\mathrm{H} \beta$ should be significantly broader than a dust-scattered $\mathrm{H} \beta$; the redshift of $\mathrm{H} \beta$ in the nucleus would result if the scattering electrons were in an outflowing wind.

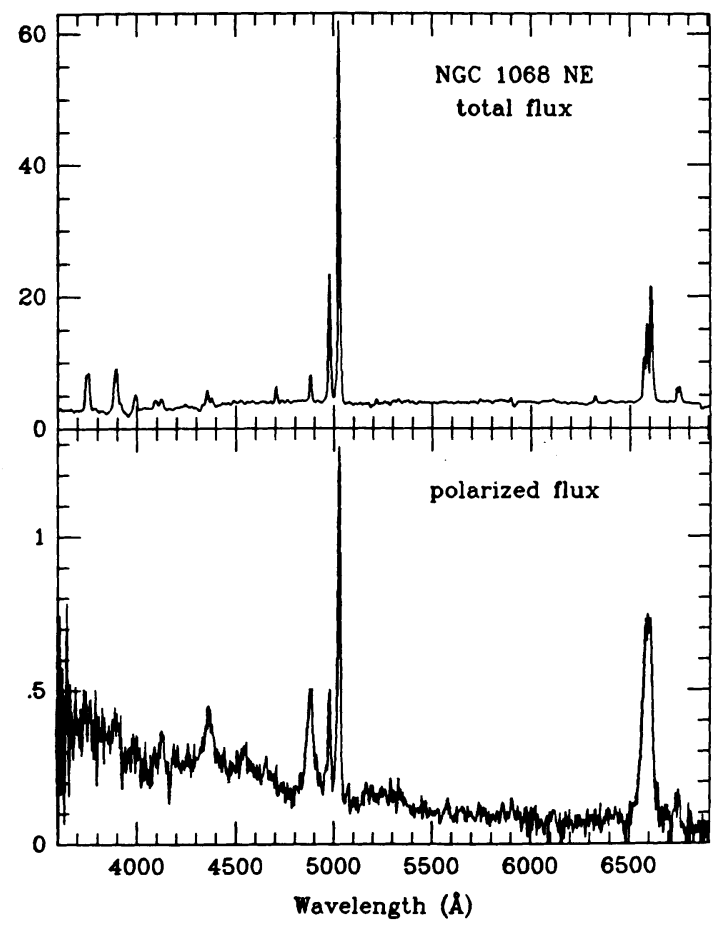

Figure 2 The total flux spectrum and polarized flux spectrum of the "north-east knot" in NGC 1068.

Further confirmation of this picture comes from x-ray data for NGC 1068. We (Antonucci and Miller 1985) pointed out that if the $\mathrm{x}$-rays detected for the nucleus by the Einstein Observatory were scattered in the same way as the broad $\mathrm{H} \beta$, then the ratio of the x-ray flux to that of broad $\mathrm{H} \beta$ is typical of what is observed for Seyfert 1 galaxies. Thus NGC 1068 behaves like a Seyfert 1 in the $\mathrm{x}$-ray region as well. Krolik and Kallman (1987) took this one step further. They predicted that the region producing the electron scattering of the $\mathrm{x}$-rays should also produce a large amount of $\mathrm{Fe} \mathrm{K} \alpha$ line 
radiation by fluorescence. Indeed data from Ginga (Koyama, this volume) does show a feature at the expected energy and flux. This result is a striking additional confirmation of the obscuring torus picture, and as we shall see, provides strong constraints on models for the scattering region.

Mathews has done an extensive set of calculations aimed at producing a selfconsistent model of the electron scattering region which must account for all of the following items:

1) The observed percent polarization $(\sim 16 \%)$.

2) The observed profile of scattered $\mathrm{H} \beta$.

3) Optically thin to x-rays

4) Have the right physical conditions for the broad line clouds.

5) Have a reasonable overall energy budget.

These five items represent challenging constraints. Mathews has explored scattering in two types of winds flowing out in a conical region: one with $n_{e} \sim r^{-2}$ and the second with $n_{e}$ a constant. The polarization requires that our viewing angle be slightly larger than the opening angle of the cone, as higher angles would produce too high a polarization. The line profile of scattered $\mathrm{H} \beta$ indicates an electron temperature significantly less than $10^{5 \circ} \mathrm{K}$, but the gas must be extremely highly ionized to have a reasonable electron scattering optical depth $(\sim 0.1)$, and be transparent to $\mathrm{x}$-rays. This in turn requires a very high ionization parameter, which must result from a high luminosity of the central object. To keep the temperature low, Mathews found it necessary to include some Compton cooling by infrared photons. Of course the input spectrum is not well known, so assumptions must be made about it. The most difficult condition to satisfy is optical thinness to soft $\mathrm{x}$-rays, but there is some evidence that the soft $\mathrm{x}$-rays may come from an entirely different region. If this is true, it is possible to construct fairly reasonable models for the scattering region.

It is natural to ask whether NGC 1068 is unique: do other Seyfert 2 nuclei have an obscured type 1 region? We now have data for 10 polarized Seyfert 2 galaxies and 6 of these - NGC 1068, Mrk 3, Mrk 348, Mrk 463 E, NGC 591, and NGC 7674 - show a broad line region in their polarized flux spectrum. As an example, Figure 3 shows the total flux spectrum and polarized flux spectra of Mrk 463E. Note the broad Balmer lines and the absence or weakness of Fe II emission. The hidden region of Mrk 348 differs in some ways from that of NGC 1068, and in fact our data (Miller and Goodrich, in preparation) show considerable variety in the characteristics of the broad line regions of Seyfert 2's.

One mystery is the absence of Seyfert 2 galaxies with very high polarization ( $\gg 20 \%$ ). Calculations by Goodrich (unpublished) show that, for a picture in which the obscuring torus is randomly oriented in space and the opening angle of the visibility is less than $60^{\circ}$, the most likely polarizations to be found are high $(\sim 40 \%)$. Evidently the geometry must not be this simple, as polarizations this high have not been detected.

The data on NGC 1068 and other Seyfert 2's with obscured high density regions indicate that if we were to observe them from direction near the axis of the torus, we would infer a much higher luminosity for the central object than the one we derive from earth. A conservative estimate is that the active nucleus of NGC 1068 would appear 100 times brighter if the obscuring torus vanished. That is, it would actually be as bright as a type 1 Seyfert galaxy and might even approach quasar luminosities. Of 
course the data show that, at least in the optical, the radiation from the nucleus does not emerge isotropically, but is directed in some kind of cone-like pattern. A fundamental question is whether the torus obscures by dust absorption, in which case it will act as a flux converter, or by atomic processes. The observation by DePoy (1988) that shows Brackett $\alpha$ in NGC 1068 at the same width as the narrow $\mathrm{H} \beta$ indicates dust must have a visual extinction greater than $100 \mathrm{mag}$ if dust also hides broad $\operatorname{Br} \alpha$, which is of course possible. An indicator of dust obscuration would be the detection of the converted luminosity in the infrared.

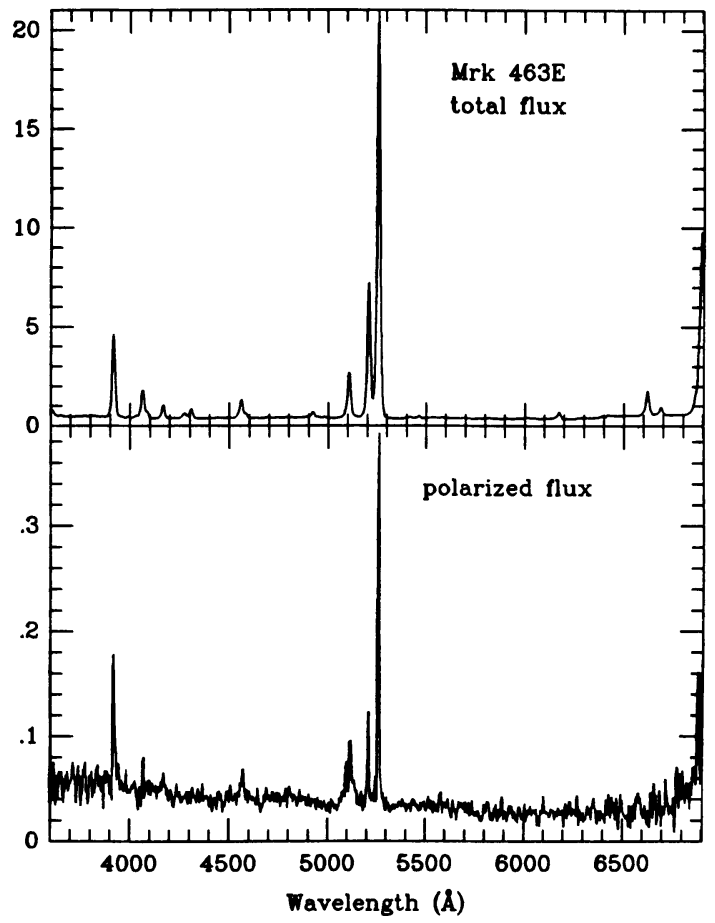

Figure 3 The total flux spectrum and polarized flux spectrum of Mrk 463E.

There are at least two other ways besides dust obscuration of achieving anisotropic or roughly collimated radiation. The first is by reflection off the inner surface of the torus. Madau (1988) has presented calculations of the structure of a thick, radiationsupported torus which does just this; the inner "funnel" acts like a mirror and collimates the light from the central region. However, whether such structures are dynamically stable and produce the requisite output spectrum at all frequencies are open questions. A third mechanism which produces anisotropic radiation is relativistic beaming, as has been proposed for BL Lacertae objects by Blandford, Rees, and others. Of course $\mathrm{H} \beta$ would not be beamed by this process, and the equivalent width of broad H $\beta$ in NGC 1068 is more or less normal for Seyfert 1's, so at most only mild 
relativistic beaming could be taking place.

Considerable other evidence has been presented at this Symposium that anisotropic radiation may be common in active nuclei (see, for example, the papers by Pogge and McCarthy). At present the data suggest for a number of cases that the radiation is truly anisotropic, because the enormous luminosities implied by isotropic radiation and dust conversion have not been detected; this is especially true of McCarthy's objects. If this holds up, it means that present determinations of the luminosities and luminosity functions of objects must be reconsidered.

\subsection{SEYFERT 1 GALAXIES}

Seyfert 1 nuclei have not turned out to be as rewarding in polarimetric studies as Seyfert 2 galaxies. As a group they tend to have low polarizations $(\sim 1 \%)$ when polarization can be detected. This polarization can have a variety of origins, as Schmidt and Miller $(1980 ; 1985)$ and Goodrich (this volume) point out. As was stated above, intrinsically polarized Seyfert 1's have their optical polarization position angles aligned with the radio structure, analogous to the behavior of quasars. We proposed (Miller and Antonucci 1983) that this could arise if Seyfert 1 nuclei were surrounded by a thin disk. The thinness of the disk makes it unlikely that our direct view of the central object is blocked, and scattering of light by material above and below the disk, but near it, would produce the parallel polarization. If the featureless continuum is produced by the disk itself, one would expect scattering in the "atmosphere" of the disk would produce polarization parallel to the surface of the disk, the opposite of what is observed if we identify the radio structure direction with the axis of the disk; models of thermal emission from the disk must account for this result.

The discovery that NGC 1068 would appear as a Seyfert 1 galaxy from other viewing directions raises the possibility that there is only one type of Seyfert galaxy, and the classification of any particular object depends solely on viewing direction. This will be the subject of the next section.

\section{One Type of Seyfert Galaxy?}

There is no question that if some Seyfert 2 galaxies, those with obscured type 1 regions, were viewed from other directions, they would be called Seyfert 1 galaxies. At the Atlanta meeting on active galaxies, I presented a discussion of this topic (Miller 1988) and now can give an update of it. I will summarize some of the arguments for and against the "one Seyfert type" picture.

For:

1. NGC 1068 and other Seyfert 2 galaxies would look like type 1's from other directions.

While this is a fact, only a small percentage of Seyfert 2's have been found to harbor a broad line region. But there is no strong evidence that these objects are different from Seyfert 2's in general, so this may be a universal property of 2's whose detectability depends on an appropriate scattering region "mirror." In further support of this is the recent suggestion that Seyfert 2's outnumber 1's by perhaps as much as $4: 1$, or even more 6:1 (Osterbrock and Shaw 1988; Khachikian, this Symposium). If the open- 
ing angle of the cone of visibility of the broad line region is $30^{\circ}$ or so, these numbers are roughly what would be expected if all Seyfert nuclei were the same.

2. The narrow line regions of 1's and 2's are the same.

Numerous studies have shown that the NLR of Seyfert 1's and 2's show the same general line spectra. Since it would be expected that the NLR would not be blocked by the obscuring torus, this result is consistent with the picture that they appear the same because they are the same thing.

Against:

1. Different radio properties

Since the radio luminosity of a Seyfert galaxy should be aspect-independent, the different average radio luminosities (Meurs and Wilson 1984) and different ratios of radio to $\mathrm{H} \beta$ luminosities (Lawrence and Elvis 1982) of type 1's and 2's are evidence of intrinsic differences between these two types. However, as Lawrence (1987) has pointed out, the galaxies involved in these studies were predominantly from the Markarian lists, and the differences could be entirely a result of selection effects.

2. Unpolarized featureless continua

If the featureless continua of Seyfert 2 galaxies are produced in a hidden region, then they can only be observed by scattering and thus should be significantly polarized. But featureless continua are a common feature of Seyfert 2 galaxies (Koski 1978), and available evidence suggests that they are typically not polarized.

3. Different [O III] luminosities

Dahari and DeRobertis (1988) have found that, with allowance for reddening effects, the [O III] luminosities of type 2's are on average about twice those of type 1's. Since the NLR should be unobscured by a torus, this indicates there is an intrinsic difference between 2's and 1's. However, if one argued that when a type 2 is viewed along the torus axis, so that it appears as a type 1 , the torus is of sufficient size to block half the [O III] region, that behind the torus, and the [O III] luminosities are thus lower.

4. Off-nuclear ionized gas different

Pogge (this volume) has found that the physical conditions in the gas surrounding the nucleus of type 1 and type 2 Seyferts are different. The differences are such that they cannot be explained in terms of aspect effects and must represent intrinsic differences. Of course his sample was not large, but the results appear highly significant.

5. All quasars are "type 1 's"

If one wants to maintain that quasars have basically the same structures as Seyfert galaxies, then where are the "type 2" quasars, those with obscured broad line regions? All $3 \mathrm{C}$ sources have now been identified, and there are no narrow line quasars in the sample. The counter to this is that obscured quasars would be called something else, e.g., a narrow line radio galaxy. However, the [O III] luminosities of these objects are not as high as those seen in quasars, so unless the NLR is also significantly obscured, these objects are intrinsically different from quasars. Our data for $3 \mathrm{C} 234$, an $\mathrm{N}$ galaxy with narrow lines, show that its featureless continuum is highly polarized and there is a hidden BLR. This shows that the "obscured Seyfert 1 region" phenomenon extends into the class of luminous radio galaxies, indicating that spirals and ellipticals can have similar central objects. 
Additional evidence for a thin disk for quasars is presented in our recent paper (Goodrich and Miller 1988) on OI 287, which is unique among quasars in showing relatively high, non-variable polarization $(\sim 8 \%)$ oriented parallel to the radio structure, $\mathrm{H} \beta$ with the same polarization, and very strong forbidden lines relative to other features. We interpret these data in terms of OI 287 being that rare quasar with a thin disk that is edge-on, so that the central continuum source and BLR are obscured, but the NLR isn't. We feel this result adds considerable weight to the thin disk picture, and if quasars have thin disks, why not Seyfert 1 galaxies?

To conclude this discussion, it is clear that at least some Seyfert 1 galaxies are poleon and look like Seyfert 2 galaxies from most directions. However I believe the above arguments strongly favor the picture that Seyfert 1's and 2's are not intrinsically identical objects whose classification depends on viewing direction. I favor a picture in which a range of opening angles can be present in the central disks of active galaxies, and the higher luminosity objects, the Seyfert 1's and QSOs, have rather thin disks. If Seyfert 2's outnumber 1's by a large amount, an implication of this argument would be that not all or even most Seyfert 2's have a hidden high density region; this question can be subject to a rather straightforward test (see 1 below).

While I have stated my beliefs about the Seyfert 1/Seyfert 2 difference question, the arguments are by no means conclusive and several further investigations would be of considerable value as follows:

1. If Seyfert 2 galaxies all have obscuring tori which block our direct view of the central continuum producing region (and any BLR present), then the only way we can see the featureless continuum is by scattering and it should always be significantly polarized if we see it at all. In fact, if all type 2's have obscuring tori, then most of them should have significantly polarized continua if the opening angle for direct view is not big $\left(<60^{\circ}\right)$. Available data are limited, but suggest that we always see the continuum and it is usually not polarized. A systematic survey to put this on a much better footing is underway by Laura Kay at Lick Observatory.

2. It would be valuable to have a bigger sample to study the properties of off-nucleus gas in Seyfert galaxies. Pogge's results are very interesting, but the number of objects in his study is relatively small.

3. One might expect if Seyfert 1's are more nearly pole-on they would have less apparently extended central radio sources because of foreshortening. This is a statistical test that would require a large number of objects.

4. A careful study of the radio luminosity functions of 1's and 2's, one where the selection effects are properly accounted for, would be of great value, but difficult.

5. Do the luminosities (or other properties) of the NLR differ in 1's and 2's? This is still uncertain, but data taken for other purposes (e.g., redshift surveys) might already exist that could address this matter.

Regardless of the outcome of these various tests it is clear that aspect effects can be important in determining what we see in active galaxies, and this should be kept in mind. For example, there already exists (see the papers by Sun and Netzer in this volume, for example) calculations of the shape of the continua expected to be observed for various orientations of a radiating accretion disk. 


\section{Summary and Conclusions}

1. There is now considerable evidence that there are systematic relationships among the characteristics of the line spectra, continuum polarization, and radio structure of active galaxies and QSOs. These relationships must all have their origin in the nature of the central objects themselves and the interaction of the central object with the surrounding galaxies.

2. Polarization studies of Seyfert 2's give strong evidence for obscuration of the innermost regions, and the presence of a thick obscuring torus is the simplest interpretation of this.

3. Available evidence suggests that Seyfert 1's and QSOs have thin disks.

4. The central disks are not necessarily aligned with the galaxy symmetry directions (not discussed in this paper).

5. Seyfert 2 galaxies and radio galaxies with a perpendicular relation between optical polarization and radio structure have similar central objects, even though the Seyferts in general are spiral galaxies while the radio galaxies are ellipticals.

6. Collimated continuum radiation from the central object is common, which has important implications for the energetics and luminosity functions of active galaxies and QSOs.

As a final remark, I feel we may be really getting somewhere in the study of active galactic nuclei. A major indication of this is the increasingly close interaction between observation and theory. It is only recently that models of the central object could be subject to observational tests and strongly influenced by them, a sign that the subject is beginning to mature.

It is a pleasure to acknowledge the extensive assistance and valuable contributions of my colleagues in this research, R. Goodrich, L. Kay, and R. Antonucci. This research was supported in part by NSF grant AST 84-06843.

\section{REFERENCES}

Antonucci, R. R. J. 1983, Nature 303, 158.

Antonucci, R. R. J., and Miller, J. S. 1984, Ap. J. 297, 621.

Dahari, O., and DeRobertis, M. M. 1988, Ap. J. (Suppl.), 67, 249.

DePoy, D. L. 1988, in Acdtive Galactic Nuclei, eds H. R. Miller and P. I. Witta

(Springer Verlag: Berlin), p. 106.

Goodrich, R. W., and Miller, J. S. Ap. J. 331, 332.

Koski, A. T. 1978, Ap. J. 223, 56.

Krolik, J. H., and Kallman, T. R. 1987, Ap. J. (Letters), 320, L5.

Lawrence, A. 1987, Publ. A.S.P. 99, 309.

Lawrence, A., and Elvis, M. 1982, Ap. J. 256, 410.

Madau. P. 1988, Ap. J. 327, 116. 
Meurs, J. S. 1988, in Active Galactic Nuclei, eds. H. R. Miller, P. J. Witta (Springer Verlag: Berlin), p. 112.

Miller, J. S., and Antonucci, R. R. J. 1983, Ap. J. (Letters) 271, L7.

Miller, J. S., Goodrich, R. W., and Mathews, W. G. 1988, in preparation.

Miller, J. S., Robinson, L. B., and Goodrich, R. W. 1988, in Instrumentation for Ground-Based Astronomy, ed. L. B. Robinson (Springer Verlag: New York), p. 157.

Miller, J. S., Robinson, L. B., and Schmidt, G. D. 1980, Publ. A.S.P. 92, 702.

Osterbrock, D. E., and Shaw, R. A. 1988, Ap. J. 327, 89.

Schmidt, G. D., and Miller, J. S. 1980, Ap. J. 240, 759. . 1985, Ap. J. 290, 517. 